\title{
Germanica
}

\section{Der hessische Landbote von Büchner und Der europäische Landbote von Menasse - Eine Parallele}

Georg Büchner's essay The Hessian Messenger and The European Courier by Robert Menasse - A comparison

Der hessische Landbote de Büchner et Der europäische Landbote de MenasseParallèle

Julie Anne Demel

\section{OpenEdition}

\section{Journals}

Édition électronique

URL : http://journals.openedition.org/germanica/2890

DOI : 10.4000/germanica.2890

ISSN : 2107-0784

\section{Éditeur}

Université de Lille

\section{Édition imprimée}

Date de publication : 30 septembre 2015

Pagination : 27-40

ISBN : 9782913857353

ISSN : 0984-2632

\section{Référence électronique}

Julie Anne Demel, «Der hessische Landbote von Büchner und Der europäische Landbote von Menasse - Eine Parallele », Germanica [Online], 56 | 2015, Online erschienen am: 30 September 2017, abgerufen am 06 Oktober 2020. URL : http://journals.openedition.org/germanica/2890 ; DOI : https://doi.org/ 10.4000/germanica.2890 


\title{
Der hessische Landbote von Büchner und Der europäische Landbote von Menasse \\ - Eine Parallele -
}

\author{
Julie Anne DEMEL \\ Lycée Janot, Sens
}

2012 publizierte Robert Menasse das Buch Der europäische $L_{\text {Landbote }}$. Der veralterte Name Landbote irritiert - denn es handelt sich um eine Berufskategorie, die es in solcher Form nicht mehr gibt. Ein Landbote war früher jener, der die Nachrichten überbrachte. Der Titel bekommt Sinn, wenn man ihn mit Dem hessischen Landboten von Büchner vergleicht. Der Büchner Text entstand im Juli 1834, von Büchners Freund, dem Pfarrer Weidig überarbeitet, und gilt als einer der wichtigsten Aufrufe zur Rebellion gegen den Absolutismus im Großherzogtum Hessen und im Deutschland des Vormärz. Menasses Verweis auf den Büchnertext wird bei näherer Untersuchung immer deutlicher und relevanter. Auch zeitgeschichtlich ist eine Parallele von Bedeutung.

Der Büchnertext gilt als ein Aufruf zur Meinungsfreiheit und zur Abschaffung der Zensur, während Robert Menasse den unmündigen

1. - Robert Menasse, Der europäische Landbote. Die Wut der Bürger und der Friede Europas oder Warum die geschenkte Demokratie einer erkämpften weichen muss, Wien, Paul Zsolnay Verlag, 2012. 
euroskeptischen Bürger von heute skizziert, der umgekehrt in der Überflutung der Informationen zu versinken droht, darum aber nicht „mündiger“" geworden ist. Daraus ergeben sich folgende Fragen: Haben die Bürger von heute von der Geschichte gelernt oder lassen sie sich lieber bevormunden und von einer Elite an der Leine führen als selbst die Initiative zu ergreifen, und welche Diskrepanzen und Klüfte zwischen der EU-Elite und dem EU-Bürger lassen ein näheres Verständnis geradezu unmöglich erscheinen? Und schließlich: sind die „hinkende“ Verfassung und das demokratische Defizit auf die Unmündigkeit und Fahrlässigkeit der EU- Bürger zurückzuführen, die es verabsäumen, die Verantwortung für das Geschick Europas selbst in die Hand zu nehmen?

\section{Die „Botschaft" und deren Adressaten}

Da Robert Menasse den Titel Der europäische Landbote gewählt hat, lässt sich die Frage nach der Kunde aufwerfen, welche der "Landbote“ überbringt. Zunächst gilt es, den Diskurs des „Hessischen Landboten“ genauer zu analysieren. Der Text ist sowohl ein politisches Flugblatt als auch eine Predigt (Botschaft) und gliedert sich in zwei Teile. Der erste Teil, der uns am meisten interessiert, stammt von Büchner und ist von den durch die Französische Revolution verkündeten Menschenrechten und Freiheiten inspiriert, während das Ende sich an dem Gerechtigkeitssinn der Bibel orientiert und eher einer Predigt gleicht.

Interessant ist zunächst der informative Charakter des Flugblattes. Als Einleitung gibt es eine Gebrauchsanweisung und Richtlinien, wie man das Flugblatt in verschiedenen Situationen zu verwenden hat, denn „wer die Wahrheit sagt, wird gehenkt" 2 :

1. Sie müssen das Blatt sorgfältig außerhalb ihres Hauses vor der Polizei verwahren;

2. sie dürfen es nur an treue Freunde mitteilen;

3. denen, welchen sie nicht trauen wie sich selbst, dürfen sie es nur heimlich hinlegen;

4. würde das Blatt dennoch bei einem gefunden, der es gelesen hat, so muss er gestehen, dass er es eben dem Kreisrat habe bringen wollen;

5. wer das Blatt nicht gelesen hat, wenn man es bei ihm findet, der ist natürlich ohne Schuld. ${ }^{3}$

Das ist natürlich eine sarkastische Anspielung auf die Zensur und die fehlende Pressefreiheit.

2. - Georg Büchner, Lenz. Der hessische Landbote, mit einem Nachwort von Martin Greiner, Stuttgart, Philipp Reclam jun. Verlag (UB Nr 7955), S. 37-53 (Fassung von Juli 1834) (hier S. 37).

3. - Ebd. 
Heutzutage im Facebook- und Twitter-Zeitalter - mit fast unbegrenzter Meinungsfreiheit -fliegen Eilmeldungen und „Botschaften“ zensurfrei über Twitter und Facebook mit Lichtgeschwindigkeit um den Globus ${ }^{4}$. Menasse bedauert aber, dass der heutige Bürger, der mit einem Überfluss an Informationen de facto konfrontiert ist, deren Rechtsgültigkeit nicht hinterfragt.

Der Büchnertext richtet sich an die Bauern und an die Handwerker, die vom Großherzog unterdrückt wurden. Der Flugschrift gingen zwei Ereignisse voraus: Das erste war das Hambacher Fest im Mai 1832, das von der frühliberalen Opposition veranstaltet wurde, an dem aber auch die anderen oppositionellen Bewegungen vertreten waren. Angesichts der manchmal stark voneinander abweichenden Forderungen (von Deutschtümelei bis zur republikanischen Forderung nach Volkssouveränität) konnte keine Einigung erreicht werden und die Delegierten verließen Hambach ohne ein gemeinsames Programm. Nur die aktivsten Gruppen planten einen bewaffneten Aufstand, der am 30. April 1833 in dem Sturm auf die Frankfurter Konstablerwache gipfelte. Die Aktion war jedoch schlecht geplant. Die Frankfurter Bürger stellten sich auf die Seite der Obrigkeit und auch die Bauern beteiligten sich nicht an der Aktion. Dementsprechend richtete sich Der Hessische Landbote nicht nur gegen die feudalen Mächte, sondern auch gegen das begüterte Bürgertum. Als einer der ersten löste sich Büchner von bürgerlich-liberalen Positionen und denunzierte die ökonomischen Verhältnisse als fundamentale Ursache der politischen Unfreiheit. Das Pamphlet hat also einen vormarxistischen Ansatz und greift den während der Französischen Revolution häufig gebrauchten Wahlspruch: „Friede den Hütten! Krieg den Palästen! “ auf5.

Fast 180 Jahre später eine Bilanz ziehend meint jedoch Menasse, dass es ein Irrtum sei zu denken, dass sich die unterdrückten Klassen vom Joch letztendlich befreit hätten: „Nie zuvor hat eine unterdrückte Klasse durch Widerstand, Aufstände und Revolutionen selbst die Macht erobert, geschweige denn eine allgemeine Befreiung, Friede, Gerechtigkeit und wahren Rechtszustand hergestellt." ${ }^{6}$ Doch haben die mit der Zeit erstandenen Freiheiten und die Entwicklungen in Europa ein neues Selbstverständnis geschaffen:

4. - Menasse selbst verwendet Facebook und kann unmittelbar die Reaktionen seiner Freunde erfahren. So postete ein Freund von ihm in Facebook die Statusmeldung: „Die EU ist unser Untergang“, die sofort hundertmal „geliked“ wurde. Siehe R., a.a.O., S. 7 und 106.

5. - Dieser Ruf wird dem französischen Schriftsteller Nicolas Chamfort zugschrieben.

6. - R. Menasse, a.a.O., S. 105. 
Jetzt entsteht in Europa zum ersten Mal eine wirklich universale Klasse [...] Diese Klasse ist universal, weil sie sich zum ersten Mal in der Geschichte nicht soziologisch als Klasse definiert, die sich über den antagonistischen Widerspruch zu einer ,herrschenden“ Klasse definiert, während zugleich eine neue entsteht, die dann alles an sich reißt, sondern weil sie sich zusammensetzt und zusammenfindet aus allen Klassen und Schichten und Gruppen Europas, die aus ihren ganz unterschiedlichen Erfahrungen und Kenntnissen ihre Expertisen einbringen in die Bewegung der Kritik an den nationalen Systemen, der Kritik an den Aporien in der Verfasstheit der Europäischen Union und an einer Globalisierung, die eindeutig nicht die Globalisierung der Menschenrechte, sondern die des Finanzkapitals ist. ${ }^{7}$

Eine „Dynamik“ entsteht, ,die politischen Druck gleichermaßen wie wachsendes Bewusstsein produziert" ${ }^{\text {"8 }}$. Robert Menasse ist aber nicht davon überzeugt, dass die EU die Demokratie in den europäischen Staaten gefestigt hat. Was im Moment gravierend bemängelt wird, ist das demokratische Defizit zwischen der EU und dem Bürger.

\section{Der Grund für das Defizit an Demokratie}

\section{Die Diskrepanz zwischen dem Bürger und der EU-Elite}

Büchners Pamphlet richtet seine Polemik gegen den Großherzog, den Hof, die Beamtenschaft und die adligen Stützen des Regimes. Vor allem klagt er die Beamten als ausführendes Organ der großherzoglichen Regierung an: ,Wer sind denn die, welche diese Ordnung gemacht haben und die wachen, diese Ordnung zu erhalten? Das ist die Großherzogliche Regierung. Die Regierung wird gebildet von dem Großherzog und seinen obersten Beamten."9 Um seine Argumentation zu bekräftigen, führt er auch die ganze Schar der staatstragenden Diener an:

Die andern Beamten sind Männer, die von der Regierung berufen werden, um jene Ordnung in Kraft zu erhalten. Ihre Anzahl ist Legion: Staatsräte und Regierungsräte, Landräte und Kreisräte, geistliche Räte und Schulräte, Finanzräte und Forsträte usw. mit allem ihrem Heer von Sekretären usw. ${ }^{10}$

Sie haben praktisch Narrenfreiheit in der Ausübung von Macht, die ihnen von der Obrigkeit zugeteilt wird: ,,sie herrschen frei und ermahnen

\footnotetext{
7. - Ebd., S. 105-106.

8. - Ebd.

9. - G. Büchner, a.a.O., S. 39.

10. - Ebd.
} 
das Volk zur Knechtschaft."11 Um die Richtigkeit seiner Behauptung zu unterstreichen, untermalt Büchner seine Argumentation mit Statistiken wie z. B.: „Für das Ministerium des Innern und der Gerechtigkeitspflege werden bezahlt 1,110,607 Gulden."12 Daher die Unbestechlichkeit der Burokraten und ihr reines Gewissen: „Unbestechlich ist [diese Gerechtigkeit], weil sie sich gerade teuer genug bezahlen lässt, um keine Bestechung zu brauchen."13 Diese Beamten diktieren außerdem Gesetze, die in einer fremden Sprache geschrieben sind:

Dafür habt ihr einen Wust von Gesetzen, zusammengehäuft aus willkürlichen Verordnungen aller Jahrhunderte, meist geschrieben in einer fremden Sprache. Der Unsinn aller vorigen Geschlechter hat sich darin auf euch vererbt, der Druck, unter dem sie erlagen, sich auf euch fortgewälzt. ${ }^{14}$

Durch dieses undurchschaubare Gesetzeslabyrinth und den Machtapparat gilt es, die Bürger im Zaum zu halten und zu unterdrücken:

Diese Gerechtigkeit ist nur ein Mittel, euch in Ordnung zu halten, damit man euch bequemer schinde; sie spricht nach Gesetzen, die ihr nicht versteht, nach Grundsätzen, von denen ihr nichts wisst, Urteile, von denen ihr nichts begreift. ${ }^{15}$

Büchner entwirft ein sehr negatives Bild von den Beamten. Sie sind der Obrigkeit total hörig, für Geldgeschenke anfällig und beteiligen sich durch ihr gewissenloses Tun an der Unterdrückung der Bauern und Bürger:

[...] aber klagt einmal über den Diebstahl, der von Staats wegen unter dem Namen von Abgabe und Steuern jeden Tag an eurem Eigentum begangen wird; damit eine Legion unnützer Beamten sich von eurem Schweiße mästen [...], klagt über eure verlorne Menschenrechte: wo sind die Gerichtshöfe, die eure Klage annehmen, wo die Richter, die Recht sprächen? ${ }^{16}$

Büchner versucht ihnen die Ungerechtigkeit der Situation zu veranschaulichen und sie aufzurütteln.

Menasse greift den Slogan von Chamfort wieder auf und macht eine Parodie daraus. In Brüssel sind die ,geschmähten ,Paläste der

\footnotetext{
11. - Ebd.

12. - Ebd.

13. - Ebd., S. 40.

14. - Ebd., S. 39-40.

15. - Ebd., S. 40.

16. - Ebd., S 40-41.
} 
Bürokratie“"17. Er überträgt die Polemik gegen das Beamtentum auf die heutige Eu-Bürokratie:

Und warum wird das, was in jedem Staat kurz und gut ,Verwaltung“ heißt, in Diskussionen über die EU immer gleich als „,Beamtendiktatur“ oder „Bürokratenmoloch“ bezeichnet? [...] für alle Missstände, Defizite, Probleme, Widersprüche, für alle Verdrossenheit wird der europäische Beamtenapparat verantwortlich gemacht. ${ }^{18}$

Parodierend zählt Menasse die herkömmlichen Klischees des Beamten auf, der spießbürgerlich lebt und die Anordnungen pünktlich und regulierungssüchtig umsetzt, ohne sie zu hinterfragen:

Die Figur des Beamten setzt sich aus den Negativbildern aller sozialen Klassen oder Berufsstände zusammen: Der Beamte ist privilegiert und dabei weltfremd wie ein dekadenter Aristokrat; behäbig und verbohrt wie der Kleinbürger; regulierungssüchtig wie ein Arbeitnehmervertreter, dabei so arbeitsscheu wie ein Lumpenproletarier, engstirnig und dabei auf hinterhältige Weise schlau wie ein Bauer (seine heilige Schrift heißt Vorschrift) [...] Und so wie alle „Sozialschmarotzer“ hat er die fixe Idee, sich zu Lasten und auf Kosten der Steuerzahler wild zu vermehren. ${ }^{19}$

Vermutlich werden die Vorurteile gegen den klassischen Beamten auf die sogenannten „Eurokraten“ einfach übertragen. So geht aus einer Eurobarometerstudie hervor: „Hauptgrund für die ,EU-Skepsis' [...] war, dass ,weitreichende politische Entscheidungen von Beamten ohne demokratische Legitimation getroffen“ würden.“20

In seinem Essay Wirtschaft und Gesellschaft entwarf Max Weber einen positiven Bürokratiebegriff ${ }^{21}$. Der Soziologe hat die Bürokratie als Rationalisierungsprozess untersucht. Als Idealtypus der Bürokratie wird bei ihm die Behörde „mit beruflichem Verwaltungsstab“ bezeichnet. Sie besitzt einen Anteil an der legitimen Macht und verhindert die Bevorzugung oder die Benachteiligung Einzelner durch willkürliche Entscheidungen, da sich alle an eine gesetzte Ordnung, d. h. an die gleichen, rational begründeten Spielregeln, bzw. Gesetze halten müssen. Menasse kommt gleichfalls zur Schlussfolgerung, dass die „Eurokratie“ nicht ganz so negativ ist, wie es zunächst scheint:

17. - Ebd., S 17.

18. - R. Menasse, a.a.O., S. 17.

19. - Ebd., S. 18-19.

20. - Ebd., S. 20.

21. - Max Weber, Wirtschaft und Gesellschaft. Grundriss der verstehenden Soziologie, Tübingen, J.C.B. Mohr (Paul Siebeck), 1921/1972. 
Wer sind diese Bürokraten [...], für die zur Unterscheidung vom herkömmlichen Staatsbeamten ein eigener Begriff geprägt wurde „Eurokraten“ [...] Der „Brüsseler Bürokrat“ ist also ein historisch völlig neuer Beamtentypus, der erste, der nicht seinem Regenten oder seiner Regierung verpflichtet ist, und der erste, der immer wieder staatliche Bürokratie in Frage stellt und deren Regeln oder Entscheidungen gegebenenfalls korrigiert oder aufhebt. ${ }^{22}$

Der österreichische Essayist kann letztlich nur eine Lobeshymne auf die neu entdeckte Europäische Kommission singen:

Die Kommission ist eine offene und transparente Institution.

Die Brüsseler Bürokratie ist extrem schlank.

Die Brüsseler Bürokratie ist extrem sparsam und bescheiden.

Die Brüsseler Bürokratie ist unglaublich billig.

Die Beamten sind lustig. Ich traf kaum trockene und verknöcherte Menschen. ${ }^{23}$

Tatsächlich findet Menasse in den Beamten der Europäischen Kommission echte Europäer: „Sie sind [...] nämlich echte Europäer: polyglott, hochqualifiziert, aufgeklärt, verwurzelt in der Kultur ihrer Herkunft, allerdings befreit von der Irrationalität einer sogenannten nationalen Identität."24 In dieser Lobeshymne werden die Beamten von ihrem schlechten Ruf reingewaschen und sie können für das demokratische Defizit nicht zur Verantwortung gezogen werden.

\section{Die Grundlagen der heutigen Demokratie}

Die heutige Demokratie basiert auf den durch die Französische Revolution erklärten Menschen- und Bürgerrechten und hat sich im 19. Jahrhundert durch das errungene Wahlrecht und die parlamentarische Repräsentation definiert. Büchner beschreibt in seinem Text den Werdegang dieser Menschenrechte:

Im Jahr 1789 war das Volk in Frankreich müde, länger die Schindmähre seines Königs zu sein. Es erhob sich und berief Männer, denen es vertraute, und die Männer traten zusammen und sagten, ein König sei ein Mensch wie ein anderer auch, er sei nur der erste Diener im Staat, er müsse sich vor dem Volk verantworten, und wenn er sein Amt schlecht verwalte, könne er zur Strafe gezogen werden. Dann erklärten sie die Rechte des Menschen. ${ }^{25}$

22. - R. Menasse, a.a.O., S. 18 und 21.

23. - Ebd., S. 21-23.

24. - Ebd., S. 23.

25. - G. Büchner, a.a.O., S. 45-46. 
Leider wurde die errungene Freiheit sukzessiv von den Tyrannen der Revolution, von Napoleon, von Ludwig dem XVIII., Karl dem X. und schließlich von Louis Philippe betrogen und der Bürger im Zustand der Unmündigkeit erhalten:

In Deutschland und ganz Europa [...] war große Freude, als der zehnte Karl vom Thron gestürzt ward, und die unterdrückten deutschen Länder rüsteten sich zum Kampf für die Freiheit. Da ratschlagten die Fürsten, wie sie dem Grimm des Volkes entgehen sollten, und die listigen unter ihnen sagten: Lasst uns einen Teil unserer Gewalt abgeben, dass wir das übrige behalten. Und sie traten vor das Volk und sprachen: Wir wollen euch die Freiheit schenken, um die ihr kämpfen wollt. [...] und so ward Deutschland betrogen wie Frankreich. ${ }^{26}$

(Es sei daran erinnert, dass der Großherzog von Hessen mit Hinblick auf die Französische Revolution von 1789 und 1830 zwar eine Verfassung und eine konstitutionelle Monarchie offiziell bekundet hatte, sich jedoch daran nicht hielt.)

Laut dem österreichischen Verfassungsrechtler Hans Kelsen „,[setzt aber] Demokratie den gebildeten Citoyen voraus“27. Das Wort citoyen bezeichnet den Bürger bzw. Staatsbürger, der in der Tradition und im Geist der Aufklärung aktiv und eigenverantwortlich am Gemeinwesen teilnimmt und dieses mitgestaltet:

[...] wenn jeder Bürger nur durch alle anderen etwas ist oder vermag, und wenn die erlangte Kraft des Ganzen der Summe der natürlichen Kräfte aller Individuen gleich ist oder sie übertrifft, erst dann kann man sagen, dass sich die Gesetzgebung auf dem höchsten Punkt der Vollkommenheit befindet, den sie zu erreichen imstande ist. ${ }^{28}$

Im Gegensatz zum „Bourgeois“, der sich durch den Besitz definiert und vor allem seinem gewerblichen Interessen nachgeht, ist der „Citoyen" ein politisch aktiver Staatsbürger, der seine Rechte und Pflichten wahrnimmt und seine politischen Rechte gerade nicht als „Geschenk“ erhält, sondern „erkämpft“.

Neben dem Wahlrecht und der repräsentativen Demokratie hat vor allem das nationale Gedankengut die Identität des deutschen Bürgers nach der 1848er Revolution geformt. Dieses Übermaß an nationalem Stolz hat letztlich 1871 zur deutschen Einigung geführt und zu den zwei Weltkriegen. Nach dem Zweiten Weltkrieg haben die Gründerväter die

26. - Ebd., S. 47.

27. - Ebd., S. 42, wo Menasse Kelsen zitiert.

28. - Jean-Jacques Rousseau, Der Gesellschaftsvertrag oder die Grundsätze des Staatsrechts, http://www.humboldt-studienzentrum.de/typo3/uploads/media/Rousseau_ Contrat_social_01pdf, Kapitel 2.7, S. 18. 
hervorragende Idee gehabt, diese nationale explosive Ladung durch eine komplexe, verflochtene Konstruktion zu entschärfen:

Die Nationen - das war nun die Idee der Gründerväter des europäischen Friedensprojekts - müssten institutionell und ökonomisch so verflochten und in wechselseitige Dependenz gebracht werden, dass das Verfolgen jeglichen Eigeninteresses gar nicht mehr anders als in gemeinschaftlichem Handel möglich ist. ${ }^{29}$

Menasse bezeichnet die Nation als das Bindeglied zwischen Europa und seinen Bürgern: „Unsere Demokratie, unsere Vorstellungen von Demokratie, unsere Erfahrungen mit Demokratie, unsere Erwartungen an Demokratie, alles, was wir für wünschenswerte demokratische Standards erachten, all dies war und ist immer nationale Demokratie.“30 Doch, was im 19. Jahrhundert als zusammenführendes Element galt, wirkt jetzt lähmend: „Nationale Demokratie blockiert die nachnationale Entwicklung, nachnationale Entwicklung zerstört Demokratie.“31

Beunruhigend ist auch die Entwicklung, nach der heute Deutschland als einzige treibende Kraft in Europa den Ton angibt. Hatte Deutschland als Auslöser des Zweiten Weltkrieges lange Zeit sich in Zurückhaltung geübt, so strotzt es jetzt wieder vor Selbstbewusstsein: „So viel Nationalismus leisteten sich Deutsche schon lange nicht mehr - eine Haltung, die doch, gemäß der Legitimationsideologien der EU, im Zuge des Zusammenwachsens Europas nach und nach verschwinden sollte."32 Tatsächlich scheinen heutzutage die Begriffe Nation, Demokratie und parlamentarische Repräsentation ineinander zu verschwimmen, und man hat keine klare Auffassung davon, was Demokratie eigentlich sein soll. In der europäischen Konstruktion scheint sich der Demokratiebegriff aufzulösen: „Kann es sein, dass die Demokratie, so wie wir sie nach 1945 mühsam und wahrlich nicht in Idealform gelernt haben und wie wir sie gewohnt sind, auf supranationaler Ebene gar nicht funktionieren kann [...]?“33 Das viel beklagte europäische Demokratiedefizit rührt daher, dass wir einen unangemessenen Begriff von Demokratie haben, dem noch die historisch gewachsene Verbindung von Nationalstaat und parlamentarischer Repräsentation zugrunde liegt.

29. - R. Menasse, a.a.O., S. 8-9.

30. - Ebd., S. 97.

31. - Ebd., S. 98.

32. - Ebd., S. 31.

33. - Ebd., S. 33. 


\section{Die ,hinkende“ Verfasstheit, bzw. Verfassung Der „Präfixburger“}

Ist die aktuelle Konstruktion an sich, also die hinkende Verfasstheit Europas für die Euroskepsis der Bürger verantwortlich, oder liegt diese an der Unmündigkeit des Bürgers, die es zu korrigieren gilt? Hat der Bürger es bis dato versäumt, die Geschicke Europas selbst in die Hand zu nehmen? Wie Büchner will Menasse den Leser aufrütteln und hinterfragt den Grundslogan der Europäischen Union als „Europa der Bürger":

Was bedeutet nun diese mit Theaterdonnerhall begleitete Forderung, aus dem bürgerlichen Europa „ein Europa der Bürger“ zu machen, was bedeutet dieses aufgeregt medienflankierte Aufstampfen der „Wut-“ oder „Mutbürger"? 34

Der Ausdruck „bürgerliches Europa“ verweist auf eine passive kleinbürgerliche Haltung, die mutlos die Instruktionen von oben befolgt, während der Ausdruck „das Europa der Bürger“ ein tatkräftiges und aktives Einschreiten des Bürgers (ohne Präfix) voraussetzt:

In einer Zeit, in der „Bürger“ noch eine klare Bedeutung hatte, brauchte er kein Präfix, um im gesellschaftlichen Diskurs Wirksamkeit zu entfalten [...] Der Begriff „Bürger“" war komplex, aber dennoch klar. Er war komplex, weil er sich mehrfach definierte, soziologisch, ökonomisch, politisch, juristisch und moralisch. ${ }^{35}$

Der „Wutbürger“ empfindet Ressentiment gegen die Obrigkeit, bzw. die Elite, ohne die Herkunft dieses aufbrausenden Gefühls näher definieren zu können: „Wut ist daher antibürgerlich. Worauf soll der Bürger [...] wütend sein? Auf die soziale Misere? [...] Auf das System?"36 Der Widerspruch entsteht aus der Diskrepanz zwischen Sicherheit und Freiheit und Freiheit und Solidarität: „Wenn der Bürger Sicherheit will, stimmt er den Einschränkungen seiner Freiheit zu. Wenn er Freiheit will, vergisst er alle Brüderlichkeit.“ 37 Da sich der Bürger nicht zu Entscheidungen und Reaktionen bewegen lässt, bleibt nur das Unbehagen als Ressentiment übrig: „Der Bürger empfindet ein Ressentiment, also eine Wut gegen die bestehende Ordnung, kann jedoch nicht definieren,

34. - Ebd., S. 59

35. - Ebd., S. 60.

36. - Ebd., S. 60-61.

37. - Ebd., S. 61. 
woher diese kommt, worauf er wirklich wütend ist und wie er sie kanalisieren kann."38

Dem „Wutbürger“ stellt Menasse der „Mutbürger“ gegenüber. Verglichen mit der Epoche von Büchner, braucht es heute im Rechtsstaat keinen Mut mehr, seine Versammlungs-, Demonstrations- oder gar Meinungsfreiheit zu offenbaren. Der Bürger brauchte Mut im Kampf gegen die Willkür, zur Durchsetzung bürgerlicher Rechte und um seine Individualität zu entfalten. Wie steht es nun heute? Den Kampf gegen die Willkür hat er sowohl verloren als auch aufgegeben und legitimiert die Macht durch seine Haltung; die bürgerlichen Rechte hat er ,gegen Sicherheit, Privilegien und Dünkel“" eingetauscht; und die Individualität hat sich in eine uniforme Masse aufgelöst ${ }^{39}$.

Der „Präfixbürger“ rebelliert gegen schon bestehende Verhältnisse, fordert demokratische Partizipation und verachtet dabei die Politik, und er verteidigt letztlich die nationalen Interessen anstelle der regionalen Interessen. Er ist in diesem Sinne eine Art „Mann ohne Eigenschaften“, der sich der jeweiligen Situation anzupassen versucht und keine rechten Entscheidungen treffen vermag. So meint Menasse, dass ein Ausdruck wie ein „Europa der Bürger“ ein Widerspruch an sich ist. Heutzutage erlebt der Bürger eine innere Zerrissenheit. Die lokale Realität ist ihm zu nah und die europäische Realität ist ihm zu abstrakt und zu fern. Er klammert sich an die nationale Identität, die ihm das Gefühl der Zugehörigkeit gibt:

Deshalb ist in seiner demonstrierten Wut und seinem behaupteten Mut weder eine Übereinstimmung mit bürgerlichen Interessen zu finden noch eine Differenz zur bürgerlichen Gesellschaft, die grundsätzlich in Frage stellen möchte, wogegen der Präfixbürger zu rebellieren glaubt. Er rebelliert gegen seine eigene politische Repräsentation, die er national zugleich legitimiert. Er wird eines Tages wirklich Mut brauchen - wenn er sich in den Spiegel schaut. ${ }^{40}$

Menasse betreibt hier Aufklärung im kantschen Sinne, indem er dem europäischen Bürger ein Spiegelbild seiner Unmündigkeit vorhält, um ihn zu Mündigkeit und Reaktion zu bewegen.

\title{
Eine ,hinkende Verfassung““
}

Unsere aktuelle Demokratieauffassung ist also eine Verbindung von Nationalstaat und parlamentarischer Repräsentation, beides Reliquien aus den Freiheitsbewegungen des 19. Jahrhunderts. In Bezug auf den

\author{
38. - Ebd. \\ 39. - Ebd., S. 61-62. \\ 40. - Ebd., S. 65.
}


Gedanken des Gesellschaftsvertrags von Rousseau, definierte Büchner den Staat wie folgt:

Der Staat? Wohnt eine Anzahl Menschen in einem Land und es sind Verordnungen oder Gesetze vorhanden, nach denen jeder sich richten muss, so sagt man, sie bilden einen Staat. Der Staat also sind alle; die Ordner im Staate sind die Gesetze, durch welche das Wohl aller gesichert wird und die aus dem Wohl aller hervor gehen sollen. ${ }^{41}$

Menasse greift die Idee des Gesellschaftsvertrags wieder auf und überträgt sie in die heutige Zeit und auf die europäische Situation, sozusagen auf eine höhere Ebene. Für Rousseau stellt der Staat einen politischen Körper dar, in dem kein Mitglied verletzt werden kann, ohne dass das Ganze daran leidet. Ebenso kann das Ganze nicht angegriffen werden, ohne dass jedes Mitglied dies spürt. Dieses Verhältnis zwingt jedes einzelne Mitglied zum Erhalt des Ganzen. Diese Idee hat sich die Europäische Union zu eigen gemacht und zunächst auf eine wirtschaftliche Ebene übertragen, um die Mitgliedsländer an sich zu binden:

Und genau das war das geniale der Idee: durch die Verschränkung und Verflechtung der Volkswirtschaften dem Nationalismus die Wurzel zu ziehen, die verfeindeten Nationen nicht nur zu versöhnen sondern durch die Interdependenz der Nationalökonomien die Nationen ganz $\mathrm{zu}$ überwinden und dadurch wahren und dauerhaften Frieden zu schaffen. ${ }^{42}$

Wie schon erwähnt sei das lähmende Element in dieser Konstruktion der Erhalt des Nationalstaates: „Der Konstruktionsfehler besteht darin, dass die Nationen, deren Macht gebrochen werden sollte, in der Union institutionell mit Macht ausgestattet werden müssten - weil es eben Vertreter von Nationen sind, die sich zur Überwindung der Nationen zusammenfinden müssen., ${ }^{43}$ Der Fehler liegt in der verkehrten Interpretation der Gewaltenteilung in der Europäischen Union und im komplexen Zusammenspiel der verschiedenen Organe: Als Exekutive spielt die Kommission als Vertreterin von den Interessen der Gemeinschaft zugleich eine wesentliche Rolle in der Gesetzgebung, da sie das alleinige Gesetzesinitiativrecht hat. Als Organe der Legislative haben Parlament und Rat ein indirektes Initiativrecht und prüfen u. a. die Gesetzentwürfe, die ihnen von der Kommission vorgelegt werden 44 . Das Parlament hat in der Tat nur eine schwache Kontrollposition und

\footnotetext{
41. - G. Büchner, a.a.O., S. 38-39.

42. - R. Menasse, a.a.O., S. 76.

43. - Ebd., S. 49.

44. - Vgl. http://europa.eu/about-eu/institutions-bodies/european-council/index _ de.htm.
} 
ist hauptsächlich für das Budget verantwortlich. Im Europäischen Rat bündeln sich die nationalen Interessen. Menasse kreidet den Rat als Bollwerk von nationalen Interessen an, der den anderen supranationalen Institutionen (Kommission und Parlament) den Weg diktiert. Auch wenn man sich auf die gemeinsame Währung einigen konnte, so wurde doch die gemeinsame Wirtschafts-, Finanz- und Fiskalpolitik blockiert ${ }^{45}$. So hat diese Art von Gesetzgebung nicht zum Ziel, einem Gesetz, das dem Wohl aller dient, gerecht zu werden, zumal ja die europäische Gesetzgebung zum Großteil in den Nationalstaaten umgesetzt werden muss: „Was auf nationaler Ebene einfach Gesetzgebung heißt - wird im europäischen Einigungsprozess pejorativ zum Regulierungswahn."46

Menasse versucht eine Lösung zu finden. Seiner Meinung nach muss die Demokratie neu erfunden werden:

Und wir müssen eine neue Demokratie erfinden. Eine Demokratie, die nicht an die Idee des Nationalstaats gekoppelt ist. Das muss man sich klarmachen: Eine nachnationale Demokratie kann nicht dieselbe Form, dieselbe Verfasstheit haben, wie sie die nationalen Demokratien für sich entwickelt haben. ${ }^{47}$

Es gilt, einen Appell an die europäischen Bürger zu richten, die sich aktiv mit dieser Frage auseinandersetzen und eine partizipative Demokratie aktiv mitgestalten sollen. Welches ist nun die aktuelle Verfasstheit der Europäischen Union, die in einen Zustand der Entscheidungslosigkeit und Widersprüchlichkeit zu versinken droht? Tatsächlich hat man ein wirtschaftliches Europa geschaffen, doch ziert man sich, wenn es um die politische und kulturelle Integration geht, obwohl der Verfassungskonvent von 2001 einen Verfassungsvorschlag an den Tag gelegt hat, auf dessen Grundlage versucht wurde, eine europäische Verfassung aufzustellen ${ }^{48}$.

Schon Jean Monnet wollte den Nationalstaat überwinden. Durch die Schaffung einer supranationalen Institution sollten die Nationalstaaten nach und nach die Souveränitätsrechte an diese supranationale Institution abgeben. Nur so könnte das Friedensprojekt zementiert werden:

45. - R. Menasse, a.a.O., S. 57.

46. - Ebd., S. 15.

47. - Ebd., S. 98.

48. - Zu seiner Zeit kritisierte Büchner die unwirksamen Verfassungsentwürfe in Deutschland. Nach der Französischen Revolution waren über 80 Verfassungen in Europa entstanden. Jedes Fürstentum hatte eine Verfassung erlassen, um seine Macht zu festigen: ,Denn was sind die Verfassungen in Deutschland? Nichts als leeres Stroh, woraus die Fürsten die Körner für sich herausgeklopft haben.[...] Denkt an die Verfassung des Großherzogtums. - Nach den Artikeln derselben ist der Großherzog unverletzlich, heilig und unverantwortlich [...] Eine solche Verfassung ist ein elend jämmerlich Ding.“ (G. Büchner, a.a.O., S. 48). 
„Ideologisch legitimiere ich die Europäische Union als ,Friedensprojekt‘, in dem die einzelnen Staaten immer mehr mit verflochten werden und zusammen wachsen." 49 Irgendwann haben jedoch die politischen Vertreter gemerkt, dass, wenn sie weiter ihre Souveränitätsrechte an die EU abgeben, sie ihre eigene Legitimation verlieren. Ein demokratisch gewählter Vertreter hat nur eine Legitimation in seinem eigenen Land und nicht auf EU-Ebene.

Menasse verwirft dem Lissabonvertrag, dass er ja ein fauler Kompromiss und im Moment hartnäckig reformresistent sei. Der aktuelle Verfassungsvertrag sollte das Friedensprojekt bestärken, bedroht jedoch in seiner unvollständigen Form die aktuelle EU. Er plädiert für supranationale Institutionen, aber nicht für reine Supranationalität. Auf der anderen Seite ist er auch ein Verfechter der regionalen Selbstverwaltung. Denn in der Region, die durch Landschaft, Geschichte, Dialekt und Bräuche definiert ist, fühlt sich der Bürger wirklich zu Hause, der Saarländer aber fremd in Hamburg. Die nationale Identität soll geschwächt werden, Menasse will aber keinen Föderalismus nach amerikanischem Vorbild: "Doch es lässt sich ja auch eine ganz neue Form der Demokratie denken, eine nachnational verfasste, in der die Entscheidungen regional getroffen werden oder eben europäisch.“50

\section{Schluss}

Dieses schlanke Buch ist gleichsam wie der Büchnertext ein Versuch, den Zeitgeist der Epoche zu erfassen und den europäischen Traum in Worte zu fassen. Da dies ein schwieriges Unterfangen ist, musste Menasse die wesentlichen Begriffe wie Bürger, Demokratie und Repräsentation neu definieren. Anstatt sich damit zu begnügen, ein Sittengemälde zu skizzieren, hat er einen Entwurf für eine Neugestaltung des europäischen Gedankens zu zeichnen versucht. Dabei lässt er dem Leser genug Freiraum, um sich eigene Gedanken machen zu können. In Europa sieht er die Möglichkeit zu etwas ganz Neuem, und man ist nach der Lektüre von dieser Möglichkeit angetan.

49. - R. Menasse, a.a.O., S. 42.

50. - http://www.faz.net/aktuell/feuilleton/buecher/rezensionen/sachbuch/robert-menasses-der-europaeische-landbote-alles-bestens-in-der-zentrale-11912216.html. 